\title{
Cross-Cultural Adaptation, Reliability, and Validity of the Revised Korean Version of Ruminative Response Scale
}

\author{
Seonyoung Lee ${ }^{1}$ and Won $\mathrm{Kim}^{2,3 凶}$ \\ 'Department of Psychology, Korea University, Seoul, Republic of Korea \\ ${ }^{2}$ Department of Psychiatry, Seoul Paik Hospital, College of Medicine, Inje University, Seoul, Republic of Korea \\ ${ }^{3}$ Stress Research Institute, Inje University, Seoul, Republic of Korea
}

\begin{abstract}
Objective Rumination is a negative coping strategy defined as repetitive and passive focusing on negative feelings such as depression. The Ruminative Response Scale (RRS) is a widely-used instrument to measure rumination, but there is continuing argument about the construct validity of the RRS, because of probable overlap between the measurement of depression and that of rumination. The RRS-Revised (RRS-R), which removed 12 items of the RRS, is suggested as a more valid instrument for measuring rumination. Therefore, we translated RRS-R into Korean and explored the reliability, validity and factor structure in patients with major depressive disorders.

Methods Seventy-nine patients with major depressive disorder took the Korean version of RRS, RRS-R, State Trait Anxiety Inventory, Beck Depression Inventory and Penn State Worry Questionnaire. We performed exploratory factor analysis of RRS-R, and tested construct validity, internal reliability and test-retest reliability.

Results The internal and test-retest reliability of RRS-R was high. Factor analysis revealed that RRS-R is composed of two factors. 'Brooding' factor explained 56.6\% and 'Reflection' factor explained 12.5\%. RRS-R, especially 'Brooding factor', was highly correlated with other clinical symptoms such as depression, anxiety and worry.

Conclusion In this study, we find out the RRS-R is more reliable and valid than the original RRS in Korean patients with depression because the RRS-R is free from the debate about the overlap of item with BDI. We also revealed that 'Brooding' is highly correlated with depressive symptoms. RRS-R may be a useful instrument to explore the implication of 'Brooding' in depression.
\end{abstract}

Psychiatry Investig 2014;11:59-64

Key Words Depression, Rumination, Validity.

\section{INTRODUCTION}

Most people experience depressive mood and cope in various ways. The two most common coping strategies for negative emotions are problem-focused coping and emotion-focused coping. The depressive disorders are related to emotionfocused coping, especially overuse of rumination. ${ }^{1}$ People has their own consistent coping style with depressive mood, and those with a ruminative coping style experience depressive mood longer and deeper. ${ }^{2}$

Received: January 23, 2013 Revised: April 12, 2013

Accepted: April 17, 2013 Available online: January 21, 2014

$\triangle$ Correspondence: Won Kim, MD, PhD

Department of Psychiatry, Seoul Paik Hospital, College of Medicine, Inje University, 9 Mareunnae-ro, Jung-gu, Seoul 100-032, Republic of Korea

Tel: +82-2-2270-0063, Fax: +82-2-2270-0344, E-mail: phrenie@naver.com

(a) This is an Open Access article distributed under the terms of the Creative Commons Attribution Non-Commercial License (http://creativecommons.org/licenses/by$\mathrm{nc} / 3.0$ ) which permits unrestricted non-commercial use, distribution, and reproduction in any medium, provided the original work is properly cited.
Rumination can be defined as repetitive and passive focusing on the meaning and implications of negative feelings, and is overly self-focused coping to negative emotion. ${ }^{2,3} \mathrm{Ru}$ minative response to depressed mood involves thinking about how sad, apathetic, and tired one feels, wondering about the causes of one's depressive symptoms, and worrying about their implications, without doing anything constructive to relieve the symptoms or improve one's mood. ${ }^{4}$ Many studies report that rumination is important in developing and maintaining depression in both clinical and non-clinical subjects. In a study in a non-clinical sample, the participants who tend towards ruminative response become more depressed after seven weeks regardless of initial level of depression and stress response. ${ }^{5}$ Another study found that the ruminative response tendency predicts the severity of depression after one year among both clinical and non-clinical subjects. ${ }^{6}$

Rumination has a high positive association with anxiety symptoms as much as depressive symptoms, and this associ- 
ation is independent of the severity of depression. ${ }^{6}$ The ruminative tendency is associated with symptoms of generalized anxiety disorder, posttraumatic stress disorder and social anxiety disorder. ${ }^{7-9}$ Trauma-related rumination has the effect of maintaining intrusive memory and negative affect after trauma experience. ${ }^{8}$ Rumination is also related to somatic and physical symptoms. Eccleston et al. ${ }^{10}$ reported that the patients with chronic pain had more difficulty suppressing thoughts about the physical symptoms than other thoughts. Children with more rumination of negative experience tend to suffer from physical symptoms. ${ }^{11}$ Rumination was reported to mediate the relation between neuroticism - the most famous temperamental susceptibility of psychopathology - and manifesting symptoms such as anxiety and depression. ${ }^{12}$ Therefore, rumination is known to be important in developing and maintaining the psychopathology including depression and anxiety.

Among the instruments to measure those ruminative response styles, the Ruminative Response Scale (RRS) developed by Nolen-Hoesekma has been widely used. ${ }^{2}$ The RRS was originally developed as a subordinate scale of the Response Style Questionnaire (RSQ). ${ }^{5}$ The RSQ consists of 71 questions, dealing with four sorts of method of treatment such as ruminative, distractive, problem-solving, and dangerous activities. However, the 'problem-solving' subordinate scale and 'dangerous activities' subordinate scale were omitted because later study found reliability to be unsatisfactory, so the present response style questionnaire consists of the RRS and Distracting Response Scale (DRS). ${ }^{13}$ The RRS consists of 22 questions that focus on subjective feelings related to the depressed mood, symptoms, and cause and consequences of the mood, and has maintained almost the same content until now, although there were partial changes over the past few years. ${ }^{14}$

The psychometric properties of the RRS, namely, reliability and validity were supported through several studies. Especially in reliability, the high inner consistency reached from Cronbach a 0.88 to 0.92 in the several previous studies. ${ }^{1,15}$ Also, several longitudinal studies showed that there is high test-retest reliability of Cronbach a 0.75 to 0.80 , proving that the RRS measures an individual's tendency of ruminative response stably. ${ }^{3,16}$

There is, however, ongoing dispute about the factor structure of the RRS in relation to construct validity, despite many ongoing studies. If we look at the previous studies, three factors - symptom-based rumination, introspection/self-isolation, and self-blame - were abstracted in a study whose subject was a group of university students. ${ }^{17}$ However, another study whose subject was a group of patients suffering from depression showed that two factors, symptom-focused rumination and self-focused rumination, were abstracted. ${ }^{18}$

There was an increasing need to revise the RRS because of repeated results that the high association between depression and rumination resulted from the overlap of the items between RRS and BDI, the measure of depression..$^{19,20}$ So Treynor et al. ${ }^{21}$ removed 12 questions of the RRS that apparently overlapped with the BDI and analyzed the factor structure of data from non-clinical subjects. They showed two factors: R (reflection); factor that shows normal self-reflective tendency and problem-solving orientation, and B (Brooding or moody pondering); factors that shows difficulties in the process of solving problems and negative self-reflection with maladjustment, such as 'Why me?'

Both the 'Refection' factor and 'Brooding' factor are positively associated with depression in the cross-sectional view. But in longitudinal observation, the 'Reflection' factor was related with decreasing depression, and the 'Brooding' factor with increase of depression. ${ }^{21,22}$ Another study also reported that 'Brooding' subtype had more association with anxiety and depression among the subtypes of rumination, so 'Brooding' is a poor coping strategy. ${ }^{23}$

In this study, we revised the original RRS by the method of Treynor et al. ${ }^{21}$ in the Korean version, and evaluated the reliability and validity, especially construct validity. We translated the original RRS and removed 12 items that are similar to items in the BDI, administered the revised questionnaire to patients with depression, performed explorative factor analysis, and evaluate the association among anxiety, depression and worry for construct validity.

We intend to find whether the association of depression with rumination comes from a real relationship or from measurement overlap. We also want to assess the association of 'Reflection' and 'Brooding' factors with clinical symptoms. In addition, we are going to test the gender difference of ruminative tendency.

\section{METHODS}

\section{Participants}

The participants were recruited among outpatients in their first visit with a diagnosis of major depressive disorders in the Department of Psychiatry, Seoul Paik Hospital, Inje University from September 2010 to February 2011. Patients with a diagnosis and past history of psychotic disorders, bipolar disorders, substance use disorders and organic brain disease were excluded. Seventy nine participants aged from 13 to 63 filled out the questionnaire. Among them, 33 were male and 46 were female. The mean age was 38.70 in men, 35.32 in women. This study protocol was reviewed and approved by the Seoul Paik Hospital Institutional Review Board, and that written informed consent for this research project was obtained from each participant. 


\section{Measure instrument}

\section{Rumination Response Scale}

This is a questionnaire in form of a self-report that evaluates the ruminative response styles, and includes 22 questions. For this study, a licensed clinical psychologist translated into Korean the ruminative response scale developed by Nolen-Hoeksema, ${ }^{2}$ and confirmed the content of the questionnaire through debate among three researchers who have at least a master's degree. After that, a bilingual person re-translated it to English and researchers reviewed and revised into the final version. The coefficient of the internal consistency (Cronbach $\alpha$ ) of RRS was 0.92 .

\section{Rumination Response Scale-Revised}

This study used the scale of Treynor et al., ${ }^{21}$ which excludes 12 questions overlapping between the RRS and BDI. Treynor et al's study showed that RRS-R consists of two factors, R (reflection) and B (brooding) subordinate scale as the result of the factor analysis. The coefficient of the internal consistency of the R-scale and B-scale (Cronbach $\alpha$ ) were 0.72 and 0.77 . The test-retest reliability measured during 2 years were 0.60 and 0.62 .

\section{Penn State Worry Questionnaire}

This is a questionnaire in form of self-report developed by Meyer et al., ${ }^{24}$ and consists of 16 questions which measure the symptom of worry. The Korean version was standardized by Kim and Min..$^{25}$ The coefficient of the internal consistency (Cronbach $\alpha$ ) of the Korean version was 0.89 .

\section{Procedure}

We first removed 12 questions (no.1, 2, 3, 4, 6, 8, 9, 14, 17,
$18,19,22)$ that overlapped with the BDI in terms of content as the previous study, ${ }^{21}$ and performed exploratory factor analysis using the principal component analysis and obliminal rotation. We then performed the internal reliability and test-retest reliability test.

For construct validity, we calculated Pearson's correlation test among the total points, subordinate scale points, and scale points of depression, anxiety, worry and physical symptoms. The correlation between the original RRS and those related scales above was also estimated and compared with the result of RRS-R. Finally, we closely examined the difference in the total points and subordinate points of the RRS and RRS-R according to gender.

SPSS 12.0 for Windows (SPSS V12.0K, SPSS Inc., Chicago, IL, USA) was used in analyzing the technical statistics, correlation and exploratory factors of the data.

\section{RESULTS}

\section{Exploratory factor analysis}

On the ground of the result from the previous study, ${ }^{21}$ we abstracted factors by principal component analysis and obliminal rotation from the revised ruminative response scale (RSS-R) which removed 12 questions among 22 questions of the RRS. We found that a two factors structure model is suitable for the factor coefficient matrix, and the total variance that those two factors explain was $69.12 \%$. 'Brooding' factor explain $56.6 \%$ and 'Reflection' factor, $12.5 \%$ of variance (Table 1). So we found the Korean version RSS-R has good construct validity.

In examining specific factors, our result was consistent with the result from the factor analysis by Treynor et al. ${ }^{21}$ overall. However, there were different findings in details. The item

Table 1. Factor structure obtained from a factor analysis of the RRS-R $(N=79)$

\begin{tabular}{|c|c|c|c|}
\hline \multirow{2}{*}{ Item } & \multicolumn{3}{|l|}{ Factor } \\
\hline & Item & 1 & 2 \\
\hline 15 & Think "why do I have problems other people don’t have?" & 0.932 & \\
\hline 10 & Think “why do I always react this way?" & 0.931 & \\
\hline 13 & Think about a recent situation, wishing it had gone better & 0.799 & \\
\hline 5 & Think "what am I doing to deserve this?" & 0.656 & \\
\hline 20 & Analyze your personality to try to understand why you are depressed & 0.570 & \\
\hline 12 & Write down what you are thinking and analyze it & & 0.914 \\
\hline 21 & Go someplace alone to think about your feelings & & 0.646 \\
\hline 11 & Go away by yourself and think about why you feel this way & & 0.527 \\
\hline Eigenvalues & & 4.528 & 1.002 \\
\hline$\%$ of variance & & 56.6 & 12.522 \\
\hline Cumulative \% & & 56.6 & 69.122 \\
\hline Cronbach $a$ & & 0.728 & 0.64 \\
\hline
\end{tabular}

Principle components analysis, obliminal rotation. RRS-R: ruminative response scale-revised, Factor 1: brooding, Factor 2: reflection 
no.16 was included in 'Brooding' factor and no. 7 was in 'Reflection' factor in the previous study, but both items were excluded from each subordinate factor in our analysis because the factor loading for both items is not different. Item No.20 (Analyze your personality to try to understand why you are depressed) was in the 'Reflection' factor in the previous study, but it was in the 'Brooding' factor in our study (Table 1).

\section{Reliability of the RRS-R}

The internal consistency of the overall scale of the RRS-R (Cronbach $\alpha$ ) was $0.89(\mathrm{n}=79)$, and the internal consistency of the 'Brooding' and 'Reflection' subordinate scale were 0.73 and $0.64(\mathrm{n}=79)$ respectively.

For the test-retest reliability, 22 participants out of 79 participants took the scale after one month. The test-retest reliability coefficient of the RRS- $\mathrm{R}$ was $0.48(\mathrm{n}=22, \mathrm{p}<0.05)$. The test-retest reliability coefficient of the 'Reflection' subordinate scale was $0.54(\mathrm{n}=22, \mathrm{p}<0.05)$ similar to the result of the previous study. ${ }^{21}$ However, the coefficient of 'Brooding' subordinate scale was 0.41 , a little less test-retest reliability.

Meanwhile, the test-retest reliability of the original RRS was low, unlike the RRS-R ( $r=0.33, n=22$, ns). These results may suggest that the RRS-R is more stable than the original RRS in measuring the cognitive styles that respond to depression.

\section{Construct validity (correlation with clinical symptoms)}

In order to examine the construct validity of the RRS-R, we examined the correlation among the total scores and two subordinate scale scores of the RRS-R, the total scores of RRS, BDI, STAI-S, STAI-T and PSWQ (Table 2). First, the total scores and two subordinate scale scores of the RRS-R showed high correlation with the total scores of the RRS, proving that the RRS-R can be used instead of the RRS.

The total scores of the RRS-R and RRS showed significant positive correlation with $\mathrm{BDI}(\mathrm{r}=0.62, \mathrm{p}<0.001 ; \mathrm{r}=0.60$, $\mathrm{p}<$ 0.001 ). This suggests that the correlation between rumination and depression is real and is not caused by the overlap of items between two measurements, because RRS-R is made by excluding the potential overlapping items. In detail, the 'Brooding factor had a higher association with depression than the 'Reflection' factor $(\mathrm{r}=0.62, \mathrm{p}<0.001 ; \mathrm{r}=0.41, \mathrm{p}<0.001)$.

With STAI-S, the RRS-R and RRS were significantly correlated $(\mathrm{r}=0.60, \mathrm{p}<0.05$ and $\mathrm{r}=0.65, \mathrm{p}<0.01)$. The 'Brooding' factor also had a stronger association with state anxiety than the 'Reflection' factor $(r=0.65, \mathrm{p}<0.01$ and $\mathrm{r}=0.43, \mathrm{p}<0.01)$. With STAI-T, they showed the similar patterns $(r=0.73, p<0.01 ; r=$ $0.71, \mathrm{p}<0.01$ ), and the 'Brooding' factor also had the stronger association $(\mathrm{r}=0.78, \mathrm{p}<0.01$ and $\mathrm{r}=0.52, \mathrm{p}<0.01)$.

With PSWQ, the RRS-R and RRS were positively correlated $(\mathrm{r}=0.69, \mathrm{p}<0.01 ; \mathrm{r}=0.58 \mathrm{p}<0.01)$, and the 'Brooding' factor was more highly correlated with PSWQ scores than the 'Reflection' factor ( $\mathrm{r}=0.67, \mathrm{p}<0.01 ; \mathrm{r}=0.57, \mathrm{p}<0.01)$.

Regarding physical symptoms, the RRS-R and RRS showed moderate correlation with SCL- 90 ( $\mathrm{r}=0.40, \mathrm{p}<0.01 ; \mathrm{r}=0.45$, $\mathrm{p}<0.01$ ), and the 'Brooding' factor was more related to physical symptoms than the 'Reflection' factor $(\mathrm{r}=0.46, \mathrm{p}<0.01$; $\mathrm{r}=$ $0.24, \mathrm{p}<0.01$ )(Table 2).

\section{Difference by gender}

There were no significant differences between genders in the total scores of RRS-R, RRS and subordinate scales of RRS$\mathrm{R}$, as shown on Table 3 .

\section{DISCUSSION}

In this study, we translated the RRS developed by NolenHoeksema ${ }^{2}$ into Korean language and produced the Korean version of RRS-R by excluding the items that overlap with BDI, following Treynor et al. ${ }^{21}$ We evaluated the reliability and va-

Table 2. Pearson correlations among RRS-R subfactors, RRS, and measures of other clinical variables $(N=79)$

\begin{tabular}{lccccccccc}
\hline & RRS-R & B & R & RRS & BDI & SCL-90 & STAI-S & STAI-T & PSWQ \\
\hline RRS-R & & $0.923^{* *}$ & $0.893^{* *}$ & $0.777^{* *}$ & $0.590^{* *}$ & $0.397^{* *}$ & $0.603^{* *}$ & $0.727^{* *}$ & $0.685^{* *}$ \\
B & $0.923^{* *}$ & & $0.651^{* *}$ & $0.750^{* *}$ & $0.648^{* *}$ & $0.464^{* *}$ & $0.648^{* *}$ & $0.781^{* *}$ & $0.668^{* *}$ \\
R & $0.893^{* *}$ & $0.651^{* *}$ & & $0.655^{* *}$ & $0.405^{* *}$ & $0.240^{*}$ & $0.430^{* *}$ & $0.520^{* *}$ & $0.567^{* *}$ \\
RRS & $0.777^{* *}$ & $0.750^{* *}$ & $0.655^{* *}$ & & $0.596^{* *}$ & $0.449^{* *}$ & $0.646^{* *}$ & $0.712^{* *}$ & $0.580^{* *}$ \\
BDI & $0.590^{* *}$ & $0.648^{* *}$ & $0.405^{* *}$ & $0.596^{* *}$ & & $0.705^{* *}$ & $0.789^{* *}$ & $0.805^{* *}$ & $0.591^{* *}$ \\
SCL-90 & $0.397^{* *}$ & $0.464^{* *}$ & $0.240^{*}$ & $0.449^{* *}$ & $0.705^{* *}$ & & $0.633^{* *}$ & $0.539^{* *}$ & $0.445^{* *}$ \\
STAI-S & $0.603^{* *}$ & $0.648^{* *}$ & $0.430^{* *}$ & $0.646^{* *}$ & $0.789^{* *}$ & $0.633^{* *}$ & & $0.800^{* *}$ & $0.665^{* *}$ \\
STAI-T & $0.727^{* *}$ & $0.781^{* *}$ & $0.520^{* *}$ & $0.712^{* *}$ & $0.732^{* *}$ & $0.539^{* *}$ & $0.806^{* *}$ & $0.633^{* *}$ \\
PSWQ & $0.685^{* *}$ & $0.668^{* *}$ & $0.567^{* *}$ & $0.580^{* *}$ & $0.591^{* *}$ & $0.445^{* *}$ & $0.665^{* *}$ & $0.712^{* *}$ &
\end{tabular}

${ }^{*} \mathrm{p}<0.05,{ }^{* *} \mathrm{p}<0.01$. RRS-R: Ruminative Response Scale-Revised, B: RRS-R factor I Brooding, R: RRS-R factor II Reflection, RRS: Ruminative Response Scale, BDI: Beck Depression Inventory, STAI: State-Trait Anxiety Inventory, PSWQ: Penn State Worry Questionnaire 
Table 3. Mean difference between male and female subjects on RRS and RRS-R subfactors

\begin{tabular}{lccc}
\hline \multirow{2}{*}{$\begin{array}{c}\text { RRS and } \\
\text { RRS-R subfactors }\end{array}$} & $\begin{array}{c}\text { Male subjects } \\
(\mathrm{N}=33)\end{array}$ & $\begin{array}{c}\text { Female subjects } \\
(\mathrm{N}=46)\end{array}$ & $\mathrm{t}$ \\
\cline { 2 - 3 } & \multicolumn{2}{c}{ Mean (SD) } \\
\hline RRS total scores & $46.46(19.40)$ & $47.48(17.46)$ & -0.245 \\
RRS-R total scores & $16.39(5.43)$ & $17.11(6.03)$ & -0.066 \\
RRS-R factor I: & $11.19(3.99)$ & $11.4(4.30)$ & -0.022 \\
brooding & & & \\
RRS-R factor II: & $5.22(1.94)$ & $5.72(2.25)$ & -0.104 \\
reflection & & & \\
\hline
\end{tabular}

There were no significant differences between genders in all parameter. RRS-R: Ruminative Response Scale-Revised, RRS: Ruminative Response Scale

lidity of this RRS-R among Korean patients with depression.

Factor analysis by principal component analysis and obliminal rotation revealed that RRS-R is composed of two factors. 'Brooding' factor explained 56.6\% and 'Reflection' factor explained $12.5 \%$. These results were consistent with those of Treynor et al., ${ }^{21}$ but the items assigned to each factor were a little different. The item 20 (Analyze your personality to try to understand why you are depressed) assigned to 'Refection' factor in Treynor et al. ${ }^{21}$ is included in the items of 'Brooding' factor in this study. Item 16 and item 7 was excluded from the subordinate scale because of low factor loading.

RRS-R and two subordinate scales (Brooding, Reflection) showed the appropriate value of Cronbach $\alpha$, good internal consistency. The test-retest reliability of RRS-R with an interval of one month is moderate. The internal consistency of the original RRS was also good, but the test-retest reliability of the original RRS is low. Although the test-retest reliability of RRS$\mathrm{R}$ is not enough reliable (0.48), it is higher than the original RRS (0.33).

This study suggests that RRS-R is more valid and reliable than the original RRS in Korean patients with depression, because the RRS-R is free from the debate about overlap with $\mathrm{BDI}$, showing better test-retest reliability.

We also tested the construct validity of RRS-R by analyzing the correlation between RRS-R and other clinical symptoms such as depression, anxiety, worry. RRS-R was highly correlated with other clinical symptoms as well as with the original RRS. Two subordinate scales of RRS-R were also correlated with other symptoms. But the 'Brooding' subscale was more highly correlated with other symptoms than the 'Reflection' subscale. This result is consistent with the study of Treynor et al. ${ }^{21}$ in that the 'Brooding' subscale was more correlated with the negative aspects of ruminative response. Another study showed that 'Brooding' was also more related to anxiety than other factors. ${ }^{23}$ Unlike RRS-R, the construct validity of the original RRS seems not to be strong, because some studies found that RRS had three factors, and only one of three correlated with depression. ${ }^{17}$ RRS-R was significantly correlated with BDI after excluding the items of RRS that potentially overlap with BDI. Therefore, we find that rumination measured by RRS-R is correlated with depression by means of their shared properties, as many studies suggested. ${ }^{20}$ We suggest that RRS-R is more valid and reliable measurement than the original RRS.

Rumination, especially 'Brooding', was highly correlated with physical complaints as well as depression, anxiety, and worry in our study. Another study reported that rumination was correlated with insomnia. ${ }^{26}$ These suggest that 'Brooding' may be a transdiagnostic process of many psychiatric problems.

The total score and two subordinate score of RRS-R were not different. The gender difference of the original RRS was not apparent in our data. This is consistent with the result of Watkins, ${ }^{23}$ but not consistent with that of Nolen-Hoeksema et al. ${ }^{27}$ Another study showed that some characteristics including the severity of depression were not different between genders. ${ }^{28,29}$ So the coping and ruminative style by gender needs further research.

There are some limitations in this study. Although one highly experienced psychiatrist diagnosed the participants with major depressive disorder, structured diagnostic measure was not used. For confirming the construct validity, confirmatory factor analysis is also helpful in addition to exploratory factor analysis. But we did only exploratory factor analysis because we did not have enough number of participants to perform both. In addition, we removed two more items out of the RRS$\mathrm{R}$ revised by Treynor et al. ${ }^{21}$ It could reduce the consistency and reliability of the measurement.

Rumination, especially 'Brooding' is one of the important components of depression. Nowadays more studies are needed to reveal the longitudinal effect of rumination to various psychopathologies. We suggest that RRS-R is a more concise and proper tool to measure rumination and is as reliable as the original RRS. We expect that more studies will be performed about the rumination using RRS-R.

\section{Acknowledgments}

This work was supported by Grant from Inje University, 2010.

The authors thank to Ho-Sung Han who helped us to collect data and reference.

\section{REFERENCES}

1. Nolen-Hoekema S. Ruminative Coping with Depression. In: Heckhausen J, Dweck CS, Editors. Motivation and Self-Regulation Across the Life Span. Cambridge: Cambridge University Press, 1998, p.237-256.

2. Nolen-Hoeksema S. Responses to depression and their effects of the duration of the episodes. J Abnorm Psychol 1991;100:569-582.

3. Nolen-Hoeksama S, Parker L, Larson J. Ruminative coping with depressed mood following loss. J Pers Soc Psychol 1994;67:92-104. 
4. Lyubomirsky S, Caldwell ND, Nolen-Hoeksema S. Effects of ruminative and distracting responses to depressed mood on retrieval of autobiographical memories. J Pers Soc Psychol 1998;75:166-177.

5. Noelen-Hoeksema S, Morrow J. A prospective study of depression and posttraumatic stress symptoms after a natural disaster: the 1989 Loma Prieta earthquake. J Pers Soc Psychol 1991;61:115-121.

6. Nolen-Hoeksema $S$. The role of rumination in depressive disorders and mixed anxiety/depressive symptoms. J Abnorm Psychol 2000;109:504511.

7. Fresco DM, Frankel AN, Mennin DS, Turk CL, Heimberg RG. Distinct and overlapping features of rumination and worry: the relationship of cognitive production to negative affective states. Cognit Ther Res 2002;26:179-188.

8. Clohessy S, Ehlers A. PTSD symptoms, response to intrusive memories, and coping in ambulance service workers. Br J Clin Psychol 1999; 38:251-265.

9. Mellings T, Alden LE. Cognitive process in social anxiety: the effects of self-focus, rumination and anticipatory processing. Behav Res Ther 2000;38:243-257.

10. Eccleston C, Crombez G, Aldrich S, Stannard C. Worry and chronic pain patients: a description and analysis of individual differences. Eur J Pain 2005;5:309-318.

11. Miers AC, Rieffe C, Meerum Terwogt M, Cowan R, Linden W. The relation between anger coping strategies, anger mood and somatic complaints in children and adolescents. J Abnorm Child Psychol 2007;35: 653-664.

12. Roelofs J, Huibers M, Peeters F, Arntz A, van Os J. Rumination and worrying as possible mediators in the relation between neuroticism and symptoms of depression and anxiety in clinically depressed individuals. Behav Res Ther 2008;46:1283-1289.

13. Luminet O. Assessment and Measurement of Rumination. In: Papageorgiou C, Wells A, Editors. Rumination: Nature, Theory, and Treatment of Negative Thinking in Depression. Chichester: Wiley, 2004, p.37-62.

14. Roelofs J, Muris P, Huibers M, Peeters F, Arntz A. On the measurement of rumination: A psychometric evaluation of the ruminative response scale and the rumination on sadness scale in undergraduates. J Behav Ther Exp Psychiatry 2006;37:299-313.

15. Just N, Alloy LB. The response styles theory and depression: tests and an extension of the theory. J Abnorm Psychol 1997;106:221-229.

16. Nolen-Hoeksema S, Davis CG. "Thanks for sharing that": ruminators and their social support network. J Pers Soc Psychol 1999;77:801-814.

17. Roberts JE, Gilboa E, Gotlib IH. Ruminative response style and vulnerability to episodes of dysphoria: gender, neuroticism, and episode duration. Cognit Ther Res 1998;22:401-423.

18. Bagby RM, Parker JDA. Relation of rumination and distraction with neuroticism and extraversion in a sample of patients with major depression. Cognit Ther Res 2001;25:91-102.

19. Conway M, Csank PA, Holm SL, Blake CK. On assessing individual differences in rumination on sadness. J Pers Assess 2000;75:405-425.

20. Segerstrom SC, Tsao JC, Alden LE, Craske MG. Worry and rumination: Repetitive thought as a concomitant and predictor of negative mood. Cognit Ther Res 2000;24:671-688.

21. Treynor W, Gonzalez R, Nolen-Hoeksema S. Rumination reconsidered: a psychometric analysis. Cognit Ther Res 2003;27:247-259.

22. Nolen-Hoeksema $S$, Wisco BE, Lyubomirsky $S$. Rethinking rumination. Pers Psychol Sci 2008;3:400-424.

23. Watkins ER. Depressive rumination and co-morbidity: evidence for brooding as a transdiagnostic process. J Ration Emot Cogn Behav Ther 2009;27:160-175.

24. Meyer TJ, Miller ML, Metzger RL, Borkovec TD. Development and validation of the Penn State Worry Questionnaire. Behav Res Ther 1990; 28:487-495.

25. Kim JW, Min BB. The Relation Among Worry, Intolerance of Uncertainty, and Problem Solving Style. Seoul: Proceedings of Annual Meeting of Korean Psychological Association; 1998.

26. Fernández-Mendoza J, Vela-Bueno A, Vgontzas AN, Ramos-Platón MJ, Olavarrieta-Bernardino S, Bixler EO, et al. Cognitive-emotional hyperarousal as a premorbid characteristic of individuals vulnerable to insomnia. Psychosom Med 2010;72:397-403.

27. Nolen-Hoeksama S, Larson J, Grayson C. Explaining the gender difference in depressive symptoms. J Pers Soc Psychol 1999;77:1061-1072.

28. Bukh JD, Bock C, Vinberg M, Gether U, Kessing LV. Gender differences among patients with a single episode. Psychopathology 2010;43:159169.

29. Joo HJ, Yeon BR, Lee KU. The impact of personality traits on emotional responses to interpersonal stress. Clin Psychopharm Neurosci 2012; 10:54-58. 